\title{
Desarrollo de competencias para mediciones con sistemas LIDAR en Latinoamérica
}

\author{
Skills development for measurements with \\ LIDAR systems in Latin America
}

\author{
René Estevan Arredondo' \\ Centro Meteorológico de Camaguey, Cuba \\ realcmw@gmail.com
}

\section{RESUMEN}

Este documento tiene el propósito de exponer las oportunidades de desarrollo de capacidades en el uso del LIDAR en las mediciones de entornos naturales y artificiales con exactitud, precisión y flexibilidad. La aplicación de los lidares en Latinoamérica en los últimos años ha venido incrementándose, y los espacios académicos para la mejora de competencias de los investigadores a través de talleres científicos también. En la actualidad, es una realidad la presencia de la Red Latinoamericana de Lidares, LALINET que está permitiendo cumplir con varios de los objetivos inicialmente propuestos. Desde el año 2001, se han venido desarrollando talleres de trabajo en el contexto latinoamericano, teniendo sus dos primeras ediciones en Camagüey en el 2001 y 2003; el tercer taller fue realizado en Popayán en el 2005; el cuarto en el 2007 en la Isla Ilhabella, Sao Paulo. Buenos Aires fue la anfitriona de la quinta edición 2009; el sexto taller tuvo como sede La Paz en el 2011; el séptimo fue celebrado en Pucón en el 2013; y el octavo en el 2015 en Cayo Coco - Cuba realizado entre los días 6 y 10 de abril con la participación de 41 asistentes y la exposición de 45 presentaciones orales y posters. Los talleres sobre lidares generan un adecuado marco para el desarrollo de competencias que permiten la aplicación de estos sistemas en diversas áreas del conocimiento, asimismo, permiten un acercamiento entre científicos y tecnólogos latinoamericanos y sus pares de otros continentes que representan a agencias gubernamentales, universidades y otros organismos.

Palabras clave: Desarrollo de capacidades, LIDAR, mediciones, lidares.

\section{ABSTRACT}

This document has the purpose to expose the opportunities for capacity-building in the use of LIDAR in the measurements of natural and artificial environments with accuracy, precision and flexibility. In recent years the application of Latin America Lidars has been increasing, also the academic areas to improve researchers skills through scientific workshops. Nowadays the LALINET, Latin America Lidar Network presence is a reality, which is allowing to achieve several initially proposed objectives. Since 2001, the workshops have been developing in the Latin American context, their first two editions were in Camaguey in 2001 and 2003; the third workshop was in Popayán 2005; the fourth in Ilhabella Island 2007, Sao Paulo. Buenos Aires hosted the fifth edition in 2009; the sixth workshop was in La Paz 2011; the seventh was in Pucon 2013; and the eighth was in Cayo Coco - Cuba 2015 between the 6th and 10th April with the participation of 41 attendees and the exposure of 45 oral presentations and posters. Workshops about Lidar generate an appropriate framework for skills development that allow the application of these systems in various knowledge areas, also to allow a approchement between Latin American scientists and technologists and their counterparts from other continents representing government agencies, universities and other organizations.

Keywords: Skills development, LIDAR measurements, Lidares.

Historial del artículo:

Recibido: 14 de mayo de 2015. Aprobado: 02 de junio 2015. Disponible en línea: 30 de junio de 2015

Dr. en Meteorología, Jefe del Grupo de Óptica Atmosférica de Camaguey - Cuba. 


\section{INTRODUCCIÓN}

Las mediciones basadas en LIDAR (acrónimo del inglés, Light Detection and Ranging) permiten a científicos y profesionales de diferentes disciplinas, entre ellas la cartografía, examinar los entornos naturales y artificiales con exactitud, precisión y flexibilidad (1), estos sistemas son empleados también para estudios atmosféricos y diversas aplicaciones. Su uso en Latinoamérica en los últimos años ha venido incrementándose, y los espacios académicos para la mejora de competencias de los investigadores a través de talleres científicos también. Así, los Talleres de Mediciones con Lidar en Latinoamérica (WLMLA, Workshop on Lidar Measurements in Latin America) congregan a diferentes grupos que emplean esta técnica, propiciando una mejor comunicación y cooperación.

Los objetivos inicialmente propuestos en estos talleres contemplaban la creación de una comunidad latinoamericana vinculada con esta técnica, propiciar el intercambio científico entre estos grupos y la creación de una Red Latinoamericana de Lidares que permitiera la planificación de investigaciones conjuntas en la región. Esta red propiciaría la estandarización de protocolos de mediciones y algoritmos, así como, la formación de capacidades en estudiantes de pre y post grado (maestrías y doctorados).

En la actualidad, es una realidad la presencia de la Red Latinoamericana de Lidares, LALINET (acrónimo del inglés, Latin American Lidar Network) que está permitiendo cumplir con varios de los objetivos

Tabla $N^{\circ}$ 1: Comunicación científica y participantes en el VIII Taller de Mediciones con Lidar en Latinoamérica, Cayo Coco, Cuba.

\begin{tabular}{|c|c|c|c|}
\hline Países & $\begin{array}{c}\text { Presentaciones } \\
\text { orales y } \\
\text { posters }\end{array}$ & Asistentes & $\%$ \\
\hline Cuba & 8 & 10 & $24,39 \%$ \\
\hline Brasil & 11 & 8 & $19,51 \%$ \\
\hline Colombia & 3 & 5 & $12,20 \%$ \\
\hline España & 11 & 5 & $12,20 \%$ \\
\hline Holanda & 2 & 3 & $7,32 \%$ \\
\hline Bolivia & 2 & 2 & $4,88 \%$ \\
\hline Perú & 0 & 2 & $4,88 \%$ \\
\hline Argentina & 3 & 1 & $2,44 \%$ \\
\hline Chile & 1 & 1 & $2,44 \%$ \\
\hline Francia & 1 & 1 & $2,44 \%$ \\
\hline Alemania & 0 & 1 & $2,44 \%$ \\
\hline Japón & 1 & 1 & $2,44 \%$ \\
\hline Estados Unidos & 1 & 1 & $2,44 \%$ \\
\hline Israel & 1 & 0 & $0,00 \%$ \\
\hline Total & 45 & 41 & $100,00 \%$ \\
\hline
\end{tabular}

inicialmente propuestos. Uno de los aportes últimos fue la medición conjunta de la erupción del volcán Calbuco en Chile (2).

Así, desde el año 2001, se han venido desarrollando talleres de trabajo en el contexto latinoamericano, teniendo sus dos primeras ediciones en Camagüey Cuba en el 2001 y 2003; la segunda edición dio inicio a los cursos sobre la técnica LIDAR, sus aplicaciones y resultados. El tercer taller fue realizado en Popayán Colombia en el 2005; el cuarto en el 2007 en la Isla Ilhabella, Sao Paulo, Brasil. Buenos Aires - Argentina fue la anfitriona de la quinta edición 2009; el sexto taller tuvo como sede La Paz - Bolivia en el 2011; el séptimo fue celebrado en la ciudad de Pucón, Chile en el 2013; y el octavo taller en el 2015 en Cayo Coco - Cuba (3).

\section{Participación y comunicación de investigaciones en lidares}

EI VIII Taller de Mediciones con Lidar en Latinoamérica (VIII WLMLA) fue realizado entre los días 6 y 10 de abril de 2015 en el Hotel Tryp Cayo Coco, Cayo Coco, Cuba; con la participación de 41 asistentes entre investigadores y estudiantes de pre y post grado, tabla $N^{\circ} 1$.

El evento permitió compartir 26 presentaciones orales y 19 posters expuestos durante las sesiones del taller. La tabla $N^{\circ} 1$ muestra la participación por países, donde se aprecia a Cuba como país sede con un 24,4 \%, luego se ubica Brasil con un 19,5\%, un aspecto importante es que progresivamente se ha visto una mayor participación de académicos y científicos de diversos países latinoamericanos, asimismo el compromiso de científicos de países europeos permitiendo un aprendizaje respecto a las múltiples aplicaciones de los lidares con énfasis en la física de la atmósfera. Es oportuno mencionar la participación de representantes de la Universidad Continental de Perú en este evento por estar ejecutando el proyecto "Estudio de la variación de las propiedades ópticas de los aerosoles debido a las quemas de biomasa para evaluar su influencia en el forzamiento radiativo terrestre" con financiamiento del gobierno peruano a través del Consejo Nacional de Ciencia, Tecnología e Innovación Tecnológica (CONCYTEC) $(4,5)$. En la Figura $\mathrm{N}^{\circ} 1$ se muestra la foto de los participantes en este evento.

Las sesiones del taller, celebradas entre el 9 y 10 de abril, estuvieron precedidas, al igual que en ediciones anteriores, por cursos sobre la técnica LIDAR dirigidos a estudiantes y jóvenes investigadores principalmente. Estos cursos divididos en niveles básico y avanzado, se llevaron a cabo entre el 6 y 7 de abril y estuvieron a cargo de científicos de reconocido prestigio a nivel internacional en sus respectivos campos de 
investigación. Las temáticas abordadas durante los cursos estuvieron relacionadas con los aerosoles, sus propiedades y efectos sobre el forzamiento radiativo, la interacción aerosoles-nubes; así como, las diversas técnicas empleadas para la medición de aerosoles, incluyendo el LIDAR. Asimismo se abordaron temas sobre los algoritmos empleados para derivar la información relacionada con los aerosoles a partir de mediciones con LIDAR, y los diferentes sistemas LIDAR existentes en la actualidad (3).

Durante las sesiones del taller (9 y 10 de abril), se expusieron trabajos relacionados con los principales tópicos de este evento, entre los que se encuentran las aplicaciones para el sensado remoto de la atmósfera, incluyendo el empleo del lidar en las ciencias medioambientales, la sinergia entre estos sistemas y otros instrumentos para estudios atmosféricos, y por último, la cooperación regional e internacional en y las nubes en la radiación solar y los procesos de transferencia radiativa que tienen lugar en la atmósfera terrestre, formaron parte de las primeras sesiones del taller. El empleo de lidares para estudios de prospección heólica, contaminación ambiental sobre ciudades de América Latina y la detección de polvo generado por tormentas en el desierto del Sahara y en el Sahel, formaron parte de estas sesiones. Una sesión especial de este evento estuvo dedicada al transporte transcontinental de contaminantes y polvo sahariano, al igual que el transporte transfronterizo de aerosoles generados a partir de la quema de biomasa y la modelación numérica del transporte de estas partículas empleando modelos numéricos de predicción como el WRF (Weather Research and Forecasting) (6). El empleo del lidar como instrumento para estudios de la capa fronteriza planetaria, determinación de las concentraciones de ozono y de contaminantes de origen antropogénico, desde diversas plataformas,

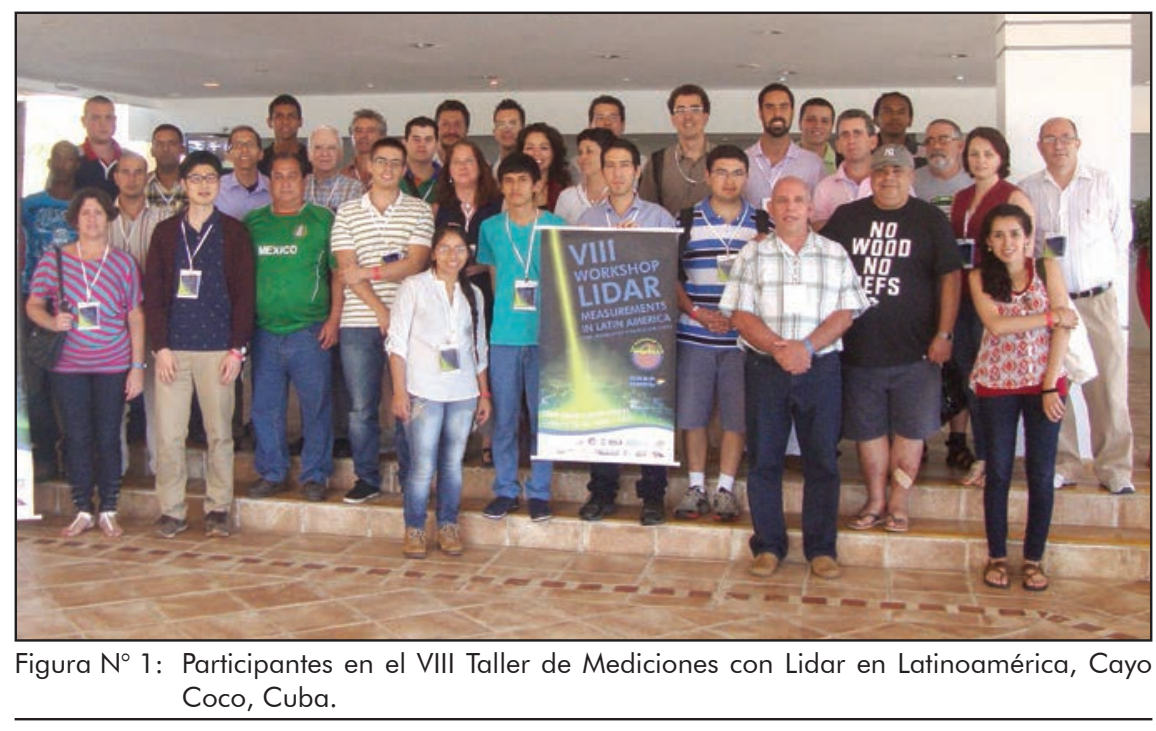

tecnología lidar y la integración en redes. Diversos fueron los trabajos presentados en las sesiones relacionadas con los tópicos del taller, destacándose entre estas la Conferencia Invitada impartida por el Prof. Patrick Hamill, de la Universidad Estatal San José, San José, California, EEUU, sobre las mediciones con lidar desde diferentes plataformas (terrestres y satelitales), utilizadas para conocer la distribución vertical de los aerosoles e identificar las diversas clasificaciones de estos, así como, la comparación de estos resultados con otros instrumentos de sensado remoto en superficie. Esta última fue una temática ampliamente abordada en las diferentes presentaciones, dada la importancia de las validaciones de las mediciones realizadas tanto con lidar como con fotómetros solares y otros instrumentos de sensado remoto, tanto activos como pasivos.

Estudios sobre el efecto que producen los aerosoles formaron parte de las presentaciones y debates en el marco de este taller.

En la actualidad existe la tendencia a la creación de redes regionales y globales que enmarquen el empleo de instrumentos, sistemas y protocolos estandarizados con el objetivo de estudiar eventos, procesos y fenómenos que afecten por igual a diversas regiones o países. En este sentido, se han creado redes regionales de lidares en Europa (EARLINET, European Aerosol Research Lidar NETwork) (7), Asia (AD-Net, Asian dust and aerosol lidar observation network) (8) y Estados Unidos (MPLNET, Micro Pulse Lidar Network) (9). América Latina no ha quedado al margen de estas tendencias, por tal motivo fue creada la Red Latinoamericana de lidares (LALINet, Latin America Lidar Network) (2), agrupando a los diversos sitios de mediciones con lidar en el continente. En estos momentos la red está abocada a la estandarización 
de los algoritmos empleados, así como, a los procesos de control de calidad del instrumental utilizado para las mediciones, realizando pruebas tales como "zero bin", "bin shift" y "dark current" entre otras. Durante el marco de este evento se realizó la reunión ejecutiva de LALINet y el fórum de discusión, donde se acordaron entre otros aspectos, la aplicación de los protocolos de medición estandarizados y las pruebas de calidad correspondientes que conduzcan a un incremento de la calidad de las mediciones realizadas y a la homogenización de la información obtenida.

\section{CONCLUSIONES}

Los talleres sobre lidares generan un adecuado marco para el desarrollo de competencias que permiten la aplicación de estos sistemas en diversas áreas del conocimiento, asimismo, permiten un acercamiento entre científicos y tecnólogos latinoamericanos y sus pares de otros continentes que representan a agencias gubernamentales, universidades y otros organismos, como es el caso de la Agencia Espacial Europea (ESA, European Space Agency) (10). Los talleres también están permitiendo encontrar nuevos cooperantes y oportunidades para hacer sostenible estos espacios académicos y científicos en la cual el común denominador es el interés de compartir visiones y experiencias de científicos para el desarrollo de un entendimiento de los requerimientos en el proceso de mediciones con lidares. Finalmente estos espacios de dialogo permiten identificar áreas clave para la cooperación con pares internacionales a nivel de conocimiento y recursos para el éxito de estudios futuros que permitirán aportes a la ciencia y tecnología.

\section{REFERENCIAS BIBLIOGRÁFICAS}

1. National Ocean Service [Internet]. Silver Spring, MD: National Oceanic and Atmospheric Administration; [Citado el 20 de mayo de 2015]. LIDAR - Light Detection and Ranging - is a remote sensing method used to examine the surface of the Earth [1 pantalla]. Disponible en: http://www. webcitation.org/6H82i1Gfx

2. LALINET [Internet]. São Paulo: Aline Network Chair and Aline Representative on Galion; [Citado el 20 de mayo de 2015]. Lalinet or Aline [1 pantalla]. Disponible en: Disponible en: http://lalinet.org

3. VIII Workshop Lidar Measurements in Latin America [Internet]. Cayo Coco; [Citado el 20 de mayo de 2015]. Disponible en: http://www.goac. cu/workshop/

4. YouTube [Internet]. Perú: Universidad Continental; [Citado el 20 de mayo de 2015]. Proyecto de investigación, Convenio CONCYTEC - Universidad
Continental [1 pantalla]. Disponible en: https:// www.youtube.com/watch? $v=$ B67qZ7xyfss

5. CONCYTEC [Internet]. Lima: Consejo Nacional de Ciencia, Tecnología e Innovación Tecnológica; [Citado el 20 de mayo de 2015]. Buscador de Proyectos [1 pantalla]. Disponible en: http:// proyectos.concytec.gob.pe/proyectos/view/ proyectos.php

6. WRF [Internet]. Boulder: National Center for Atmospheric Research; [Citado el 20 de mayo de 2015]. The Weather Research and Forecasting Model [1 pantalla]. Disponible en: http://www. wrf-model.org/

7. EARLINET [Internet]. Tito Scalo: Istituto di Metodologie per I'Analisi Ambientale; [Citado el 20 de mayo de 2015]. A European Aerosol Research Lidar Network to Establish an Aerosol Climatology: EARLINET [1 pantalla]. Disponible en: http://www.earlinet.org/

8. AD-Net [Internet]. [Citado el 20 de mayo de 2015]. AD-Net, the Asian dust and aerosol lidar observation network [1 pantalla]. Disponible en: http://www-lidar.nies.go.jp/AD-Net/

9. Network for detection of atmospheric composition change [Internet]. NOAA; [Citado el 20 de mayo de 2015]. Micro Pulse Lidar Network (MPLNET) [1 pantalla]: Disponible en: http://www.ndsc.ncep. noaa.gov/coop/mplnet/

10. European Space Agency, ESA. Disponible en: http://www.esa.int/ 